\title{
Efecto coadyuvante del extracto liofilizado de Passiflora edulis (maracuyá) en la reducción de la presión arterial en pacientes tratados con enalapril
}

\author{
Adjuvant effect of freeze-dried extract of Passiflora edulis (passion fruit) in reducing blood \\ pressure in patients treated with enalapril
}

\author{
Juan Rojas ${ }^{1}$, Sergio Ronceros ${ }^{1}$, Robert Palomino ${ }^{1}$, Marcia Salas², Rubén Azañero $^{2}$, \\ Hernán Cruz ${ }^{3}$, Agustín Rojas ${ }^{3}$, Jorge Asmat ${ }^{4}$, Juan Tam ${ }^{5}$ \\ ${ }^{1}$ Instituto de Investigaciones Clínicas, Facultad de Medicina, Universidad Nacional Mayor de San Marcos. Lima, Perú. \\ ${ }^{2}$ Hospital Nacional Dos de Mayo. Lima, Perú. \\ ${ }^{3}$ Centro de Salud de Moche, Trujillo, Perú. \\ ${ }^{4}$ Centro de Salud de Laredo, Trujillo, Perú. \\ ${ }^{5}$ Hospital Belén. Trujillo, Perú.
}

\begin{abstract}
Resumen
Objetivos: Determinar el efecto coadyuvante antihipertensivo y la seguridad del jugo del fruto de maracuyá en pacientes hipertensos en tratamiento con enalapril. Diseño: Ensayo clínico prospectivo piloto, de fase II, aleatorizado, a doble ciego, de grupos paralelos, controlado, de búsqueda de dosis y evaluación del producto. Institución: Instituto de Investigaciones Clínicas, Facultad de Medicina, UNMSM; Hospital Nacional Dos de Mayo en Lima; Hospital Belén de Trujillo y Centros de Salud de Moche y Laredo, en la ciudad de Trujillo. Participantes: Pacientes hipertensos. Intervenciones: Los pacientes fueron asignados aleatoriamente a 4 grupos. Todos recibieron enalapril $10 \mathrm{mg} /$ día y, además, el primer grupo recibió placebo y los demás 2, 3 y 4 cápsulas de $500 \mathrm{mg}$ de liofilizado de jugo de maracuyá/día, respectivamente. Principales medidas de resultados: Disminución de la presión arterial. Resultados: Los grupos que recibieron enalapril más maracuyá tuvieron una mejor reducción de la presión sanguínea en comparación con el grupo que recibió enalapril más placebo. El grupo tratado con enalapril más 4 cápsulas de jugo liofilizado de maracuyá/día produjo al final del experimento una reducción de la presión sistólica de $6,73 \mathrm{mmHg}$ y de la presión diastólica de $5,33 \mathrm{mmHg}(p<0,05)$, en comparación con el grupo enalapril más placebo. No se observó efectos adversos por el tratamiento. Conclusiones: El jugo del fruto de $P$. edulis fue coadyuvante efectivo del enalapril en la disminución de la presión arterial en pacientes con hipertensión estadio 1, y demostró ser seguro.

Palabras clave: Passiflora; presión arterial; hipertensión.
\end{abstract}

\begin{abstract}
Objectives: To determine the adjunctive antihypertensive effect and safety of passion fruit juice in patients with hypertension treated with enalapril. Design: prospective pilot clinical trial, phase II, randomized, double blind, parallel group, controlled, dose finding and evaluation of the product. Setiing: Instituto de Investigaciones Clínicas, Facultad de Medicina, UNMSM, Dos de Mayo Hospital in Lima, Hospital Belen de Trujillo and Moche and Laredo Health Centers in Trujillo city. Participants: Patients with arterial hypertension. Interventions: Patients were randomly assigned to four groups, each receiving enalapril $10 \mathrm{mg} /$ day; the first group also received placebo and the other, 2, 3 and 4 groups, $500 \mathrm{mg}$ capsules of freeze-dried passion fruit juice / day, respectively. Main outcome measures: Reduction in blood pressure. Results: Groups receiving enalapril and passion fruit had better reduction in blood pressure compared with the group receiving enalapril plus placebo. The group treated with enalapril plus 4 capsules of freezedried passion fruit juice / day had a reduction in systolic blood pressure of 6,73 $\mathrm{mmHg}$ and diastolic blood pressure of $5,33 \mathrm{mmHg}(p<0,05)$ compared with the enalapril group more placebo. No adverse effects were observed. Conclusions: $P$. edulis juice fruit was effective adjuvant to enalapril in reducing blood pressure in patients with stage 1 hypertension, with proved safety.
\end{abstract}

Key words: Passiflora; blood pressure; hypertension.

\section{INTRODUCCIÓN}

La enfermedad cardiovascular continúa siendo la causa más común de muerte en los países industrializados, siendo la hipertensión arterial el factor de riesgo tratable más frecuente. La prevalencia de hipertensión arterial en Europa es en promedio $42,2 \%$, siendo más alta en Alemania (55\%), seguido por Finlandia (49\%), España (47\%), Inglaterra (42\%), Suecia $(38 \%)$ e Italia $37 \%$, mientras que en EEUU se encontró que está en 27,6\% promedio ${ }^{(1,2)}$. En la región de las Américas, esta prevalencia se considera que oscila entre 10 y $25 \%$ de los adultos ${ }^{(3)}$.

En el Perú, se ha comunicado en 1971 una prevalencia de $16,6 \%$ a nivel del mar y de $7,4 \%$ a nivel de la altura, en adultos de 30 años a más ${ }^{(4)}$. Estudios realizados entre 1998 y 1999 en cinco ciudades del país revelaron una prevalencia de hipertensión arterial de 17,5\% en hombres y de $9,2 \%$ en mujeres ${ }^{(5)}$. En el año 2004, en un estudio realizado en 26 ciudades, la prevalencia de hipertensión en la población general peruana fue $23,7 \%$. En la costa, la prevalencia fue $27,3 \%$; en la sierra $18,8 \%$ y $22,1 \%$ en las alturas menores y mayores de $3000 \mathrm{msnm}$, respectivamente; en la selva, $22,7 \%{ }^{(6)}$.

El tratamiento de la hipertensión arterial, debido a que es una enfermedad crónica, conlleva el uso prolongado de fármacos, con diverso grado de reac- ciones adversas, que incluyen sedación, trastornos de la concentración mental, hipotensión, insuficiencia renal aguda, hipercalcemia, tos seca acompañada algunas veces de sibilancia, angioedema, entre otros ${ }^{(7)}$; por lo que es necesario la búsqueda de nuevas fuentes terapéuticas con menor riesgo, siendo el reino vegetal una alternativa.

Se ha encontrado que existen plantas con actividad antihipertensora ${ }^{(8,9)}$. Los estudios preclínicos han demostrado esta actividad en los extractos de la hoja de olivo (Olea europaea), en animales de experimentación, tanto por vía oral como intravenosa ${ }^{(10)}$; el ajo (Allium sativum) redujo la presión arterial en 
ratas hipertensas con ligadura de la arterial renal ${ }^{(11)}$; las plantas colombianas Croton schiedeanus, Curatella americana y Lupinus amandus mostraron significativa actividad antihipertensiva en ratas espontáneamente hipertensas ${ }^{(12)}$; el extracto acuoso-metanólico de las semillas de Carum copticum causó una caída de la presión arterial, dependiente de la dosis en ratas anestesiadas ${ }^{(13)}$; el extracto de la raíz de Stephaniae tetrandrae retornó a sus niveles normales la presión arterial sanguínea en ratas hipertensas por acetato de deoxicorticosterona-sal (DOCA-sal) (14); la medicina herbal japonesa Tokishakuyaku-san mejoró la hipertensión y el retardo del crecimiento intrauterino en ratas con preeclampsia inducidas por L-NAME ${ }^{(15)}$.

Los metabolitos secundarios de las plantas también han demostrado tener efecto antihipertensivo; los polifenoles del té verde y té negro atenuaron el incremento de la presión arterial sanguínea en ratas espontáneamente hipertensas ${ }^{(16)}$; el flavonoide quercetina indujo una reducción significativa de la presión arterial y la frecuencia cardiaca en ratas espontáneamente hipertensas ${ }^{(17)}$; por este mismo modelo experimental, los flavonoides obtenidos de Spergularia purpurea también exhibieron acciones antihipertensivas ${ }^{(18)}$.

Passiflora edulis Sims es una planta originaria de la amazonía brasileña, conocida con el nombre común de maracuyá, parchita, calala, maracujá, yellow passion-fruit ${ }^{(19)}$. Es una especie cultivada ampliamente en países tropicales y subtropicales y existen dos variedades: Passiflora edulis Sims var. flavicarpa, cuyos frutos son amarillos y crece desde el nivel del mar hasta los 1000 msnm; y la Passiflora edulis Sims var. purpurea, con frutos color púrpura y que se adapta a zonas altas por encima de $1200 \mathrm{msnm} .{ }^{(20)}$. La especie vegetal Passiflora edulis, comúnmente llamada 'maracuyá', es usada en medicina tradicional como relajante muscular, sedante; también en insomnio, nerviosismo, dolores estomacales, tumores intestinales, fiebre, hipertensión y diuresis ${ }^{(21,22)}$.

Los autores del presente trabajo demostraron, en un estudio preclínico previo realizado en ratas, que tanto el extracto etanólico de las hojas como el jugo de Passiflora edulis tienen efecto antihipertensivo y carece de toxicidad aguda ${ }^{(23)}$. Asimismo, no existe a la fecha ninguna comunicación en la literatura científica sobre ensayos clínicos en humanos sobre este efecto. Basados en estos antecedentes nos propusimos realizar un estudio clínico piloto para demostrar el efecto coadyuvante antihipertensivo del jugo de Passiflora edulis (maracuyá) en pacientes hipertensos en terapia combinada con enalapril y demostrar su seguridad.

\section{MÉTODOS}

El presente estudio fue un ensayo clínico prospectivo, de fase II, aleatorizado, a doble ciego, de grupos paralelos, controlado, de búsqueda de dosis y evaluación del producto. El protocolo del estudio fue aprobado por el Comité de Ética en Investigación de la Facultad de Medicina de la Universidad Nacional Mayor de San Marcos (Código № 0005/06, de fecha 14 de junio del 2006).

El fruto maduro de Passiflora edulis (maracuyá) fue colectado en la ciudad de Trujillo, Perú. Se extrajo el jugo del fruto y se pasó por un colador para separarlo de las semillas; finalmente, se filtró utilizando papel de filtro rápido con poros de 4,7-4,6 micras y se liofilizó a - $45^{\circ} \mathrm{C}$ y 100 atmósferas de presión durante 24 horas. Las cápsulas fueron formuladas para contener cada una $500 \mathrm{mg}$ de liofilizado de jugo de maracuyá y como excipientes $5 \mathrm{mg}$ de dióxido de silicio (Aerosil ${ }^{\circledR}$ ) y $35 \mathrm{mg}$ de celulosa microcristalina. Las cápsulas de placebo se formuló con 500 mg de almidón. En ambos casos, se utilizó cápsulas № 00 y del mismo color.

Participaron del ensayo 32 pacientes que asistieron al servicio de medicina del Hospital Nacional Dos de Mayo, de la ciudad de Lima, y en Trujillo al Hospital Belén, Centro de Salud de Laredo y Centro de Salud de Moche. Los pacientes fueron seleccionados cuando cumplieron con los criterios de inclusión o exclusión y se designó aleatoriamente cuatro grupos de trabajo. Los grupos estuvieron conformados por pacientes con presión sistólica de 140-159 mmHg o presión diastólica de 90-99 mmHg. Todos los grupos recibie- ron enalapril $10 \mathrm{mg} /$ día (según estándar habitual de tratamiento para hipertensión leve) y, además, el primer grupo $(n=8)$ recibió placebo en cápsulas similares a las del jugo liofilizado de maracuyá (grupo EP); los demás grupos recibieron 2, 3 y 4 cápsulas/día, respectivamente [grupos EM2 ( $n=8)$, EM3 $(n=8)$ y EM4 $(n=8)]$. El tratamiento fue por 4 semanas y la presión arterial fue registrada con el paciente sentado, luego de 5 minutos de reposo, brazo estirado a nivel del corazón; los registros fueron tomados una vez cada semana, según lo recomendado para este tipo de pacientes.

Los criterios de inclusión fueron pacientes con presión sistólica de 140$159 \mathrm{mmHg}$ o presión diastólica de 90-99 $\mathrm{mmHg}$, mayores de 18 años de edad, diagnosticados por primera vez de hipertensión arterial y sin patologías subyacentes. Los criterios de exclusión fueron pacientes con presión sistólica $\geq 160 \mathrm{mmHg}$ o presión diastólica $\geq 100$ $\mathrm{mmHg}$, diabetes mellitus, insuficiencia renal, enfermedad cerebrovascular, fumadores, e infarto de miocardio previo.

Al inicio del experimento se realizó un electrocardiograma y exámenes de laboratorio: glucosa, creatinina, colesterol total, HDL, LDL, triglicéridos y transaminasas. Se repitió los exámenes de laboratorio al final del estudio, para evaluar la seguridad del tratamiento. Se recomendó a los pacientes sujetos de estudio que no ingirieran en su dieta diaria sustancias que pudieran causar variables extrañas y variar los resultados (jugo de maracuyá, chicha morada, noni, entre otros).

Al momento de enrolarse en el estudio, los sujetos fueron informados acerca del objetivo, los procedimientos a realizarse y los métodos de medición. Todos accedieron a firmar el consentimiento informado escrito para formar parte del estudio. Los datos fueron consignados en las historias clínicas y en formatos preparados para la recolección de datos. Durante el estudio se siguió las recomendaciones de la Declaración de Helsinki de la Asociación Médica Mundial ${ }^{(24)}$ y las Buenas Prácticas Clínicas ${ }^{(25)}$.

Los resultados obtenidos de las mediciones de presión arterial fueron expresados como media aritmética \pm error 
estándar y sometidos a análisis de varianza (Anova) de un factor; las diferencias entre los grupos fueron determinadas por la prueba post hoc de Dunnett, el nivel de significancia fijado fue $p<0,05$. Los datos fueron procesados con el software estadístico SPSS 15,0 de Microsoft.

\section{RESULTADOS}

Las características generales de los pacientes que participaron en el estudio se presentan en la tabla 1. La edad media de los cuatro grupos de estudio fue 61 años con un mínimo de 43 y un máximo de 83 años, y un intervalo de confianza para la media al 95\% de 57,15 a 65,09 años.

Los valores de presión arterial tanto sistólica (PAS) como la presión arterial diastólica $(\mathrm{PAD})$ y la presión arterial media (PAM) no difirieron estadísticamente entre los grupos en la medición basal. La presión sanguínea fue disminuyendo paulatinamente, en todos los grupos, desde la primera semana y se mantuvo esa tendencia hasta el final del experimento; no obstante, en los grupos que recibieron enalapril junto a maracuyá, hubo una mejor reducción significativa de la presión arterial en comparación con el grupo que recibió enalapril más placebo. Esta reducción no fue estadísticamente significativa para el caso de PAS, a pesar que el grupo que recibió tratamiento con 4 cápsulas de maracuyá/día (grupo EM4) produjo una reducción de PAS de hasta $6,81 \mathrm{mmHg}$, en la segunda semana, culminando en $6,73 \mathrm{mmHg}$ en la cuarta semana, en comparación al placebo (grupo EP). Sin embargo, en el grupo EM4 se produjo una reducción significativa de PAD y PAM desde la segunda semana de tratamiento hasta el fin del ensayo clínico, observándose una disminución de PAD de 5,75 $\mathrm{mmHg}(p<0,05), 6,69 \mathrm{mmHg}(p<0,005)$ y $5,33 \mathrm{mmHg}(p<0,05) ; y$ de PAM de 6,10 $\mathrm{mmHg}(p<0,05), 6,12 \mathrm{mmHg}(p<0,005)$ y $5,80 \mathrm{mmHg}(p<0,05)$, en la segunda, tercera y cuarta semanas de tratamiento, respectivamente, en comparación con el grupo EP. La disminución de PAS, PAD y PAM a lo largo de las 4 semanas de tratamiento se muestra en las figuras 1,2 y 3 , respectivamente.

Los resultados de los parámetros bioquímicos en sangre realizados al finalizar
Tabla 1. Características generales de los pacientes con hipertensión estadio 1 (leve) que participaron en el estudio.

\begin{tabular}{lcccc}
\hline & \multicolumn{4}{c}{ Grupos } \\
\cline { 2 - 5 } Características & EP & $\begin{array}{c}\text { EM2 } \\
(n=8)\end{array}$ & $\begin{array}{c}\text { EM3 } \\
(n=8)\end{array}$ & $\begin{array}{c}\text { EM4 } \\
(n=8)\end{array}$ \\
\hline $\begin{array}{l}\text { Edad } \\
\quad \text { Media } \pm \text { DE }\end{array}$ & $59,3 \pm 13,7$ & $60.0 \pm 9,9$ & $61,6 \pm 9,8$ & $63,6 \pm 12$ \\
$\quad$ Rango & $43-80$ & $44-77$ & $46-77$ & $50-83$ \\
Sexo: $n(\%)$ & & & & \\
$\quad$ Masculino & $5(62,5)$ & $3(37,5)$ & $2(25,0)$ & $4(50.0)$ \\
$\quad$ Femenino & $3(37,5)$ & $5(62,5)$ & $6(75,0)$ & $4(50.0)$ \\
\hline
\end{tabular}

EP: Enalapril + placebo

EM2: Enalapril + 2 cápsulas de 500 mg de Passiflora edulis/día

EM3: Enalapril + 3 cápsulas de 500 mg de Passiflora edulis/día

EM4: Enalapril + 4 cápsulas de $500 \mathrm{mg}$ de Passiflora edulis/día el experimento no mostraron alteración en comparación con los realizados al inicio del mismo, manteniéndose dentro de los valores considerados normales. La observación clínica de los pacientes tratados puso en evidencia que en un solo caso se presentó un cuadro de diarrea leve que no motivó la suspensión del tratamiento.

\section{DISCUSIÓN}

Los resultados del presente estudio demostraron que la presión sanguínea disminuyó desde la primera semana de tratamiento hasta el final del experimento, debido a que todos los grupos recibieron enalapril, un conocido fármaco antihipertensivo que inhibe la enzima convertidora de angiotensina ${ }^{(7)}$. Sin embargo, se observó una mejor reducción de la presión sanguínea por efecto del tratamiento combinado con Passiflora edulis (maracuyá), y teniendo en cuenta que todos los grupos recibieron enalapril en la misma dosis, la reducción de la presión arterial respecto del grupo placebo se puede atribuir a efecto de $P$. edulis. Así la PAD (figura 2) y PAM (figura 3) disminuyeron significativamente desde la segunda semana de tratamiento con 4 cápsulas de $500 \mathrm{mg}$ de $P$. edulis/día (EM4), en comparación con el grupo

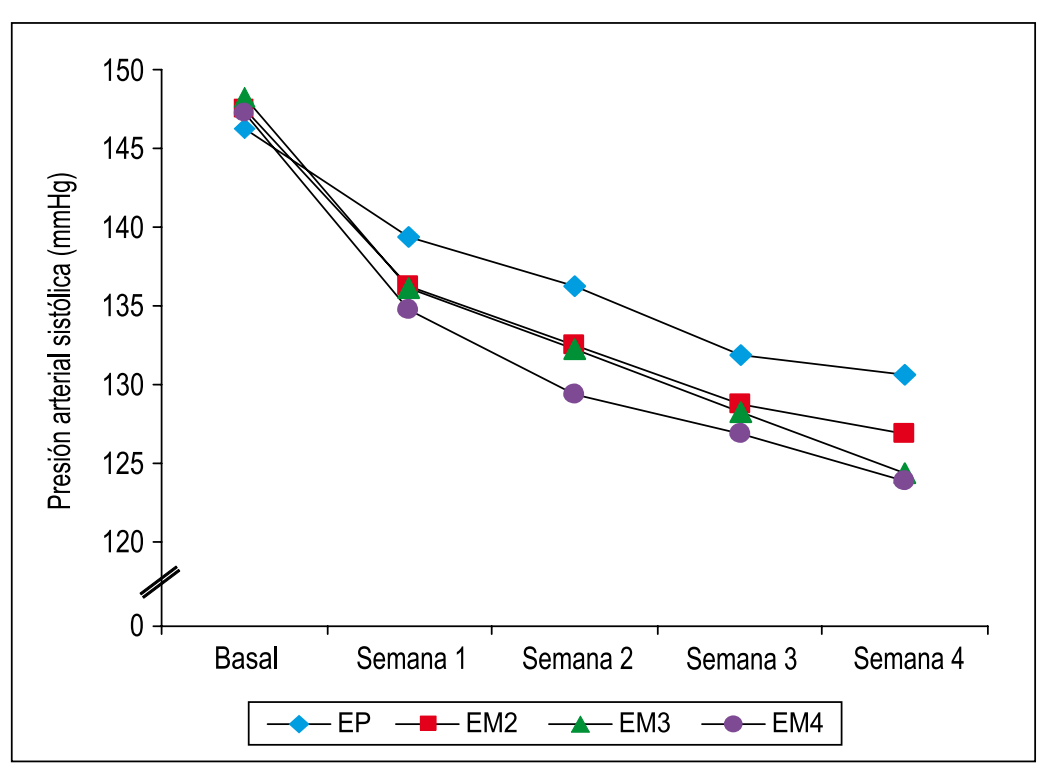

Figura 1. Efecto de $P$. edulis (maracuyá) sobre la presión arterial sistólica en pacientes hipertensos tratados con enalapril. 


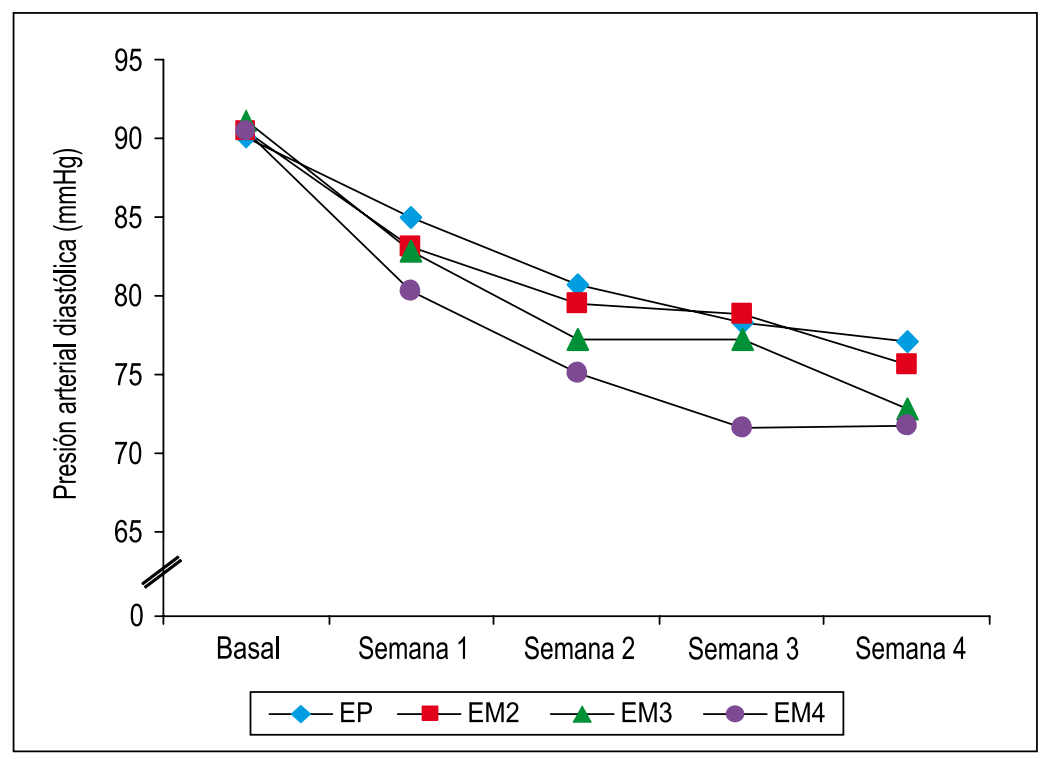

Figura 2. Efecto de $P$. edulis (maracuyá) sobre la presión arterial diastólica en pacientes hipertensos tratados con enalapril. placebo (EP), tendencia que se mantuvo hasta la culminación del estudio.

Otros estudios clínicos también han demostrado que las plantas y sus compuestos químicos pueden modular la presión arterial. En un estudio doble ciego controlado con placebo realizado en Irán, Asgary S y col., 2000, demostraron una disminución significativa de PAS y PAD por efecto del tratamiento con el extracto hidroalcohólico de Achillea wilhelmsii, lo cual fue atribuido al alto contenido de flavonoides de la planta ${ }^{(26)}$; el jugo de uva redujo significativamente la presión sistólica en $7,2 \mathrm{mmHg}$ y la presión diastólica en $6,2 \mathrm{mmHg}$ en 40 hombres coreanos hipertensos; también aquí se atribuyó a los flavonoides el mejoramiento de la función endotelial, al incrementar la producción de óxido nítrico ${ }^{(27)}$. En un estudio clínico realizado en Francia y que incluyó 1286 mujeres, se demostró que el consumo de alimentos ricos en flavonoides estuvo inversamente relacionado con la presión sanguínea sistólica ${ }^{(28)}$; la fitote-

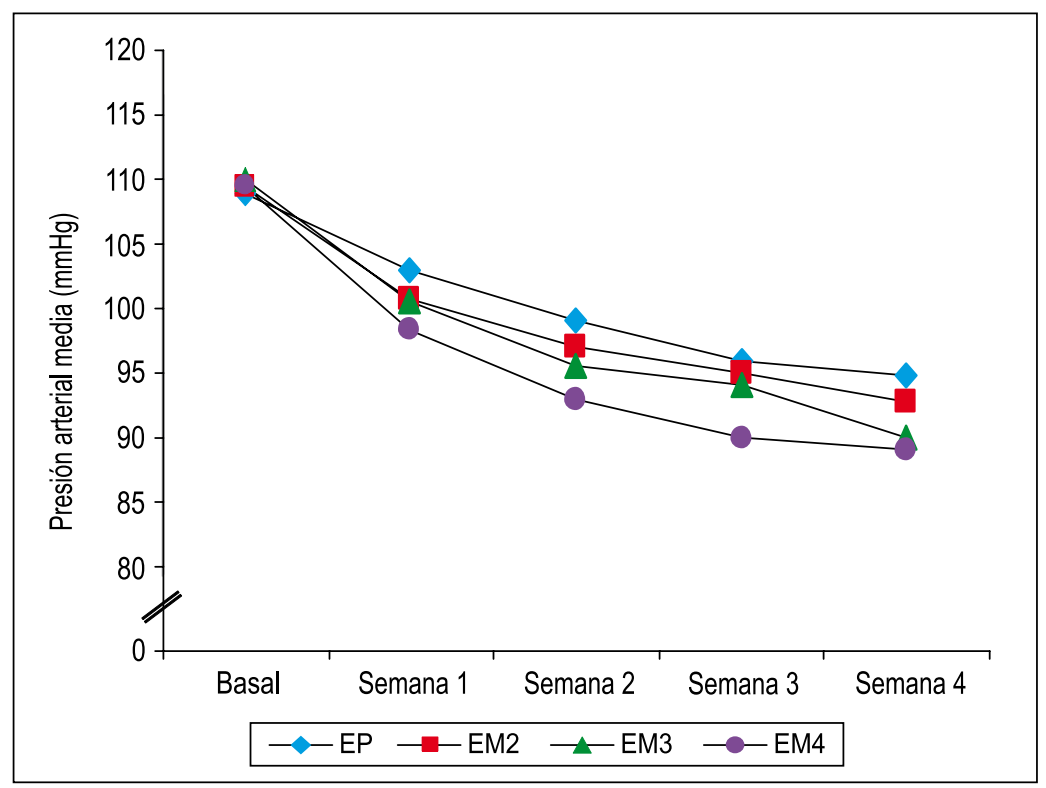

Figura 3. Efecto de $P$. edulis (maracuyá) sobre la presión arterial media en pacientes hipertensos tratados con enalapril. rapia con ajo (Allium sativum) en sujetos con hipertensión arterial primaria solo disminuyó $3 \mathrm{mmHg}$ la PAS y PAD en mujeres ${ }^{(29)}$. En un estudio con 40 gemelos monocigotos prehipertensos, el extracto de hoja olivo (Olea europea) disminuyó $6 \mathrm{mmHg}$ la PAS y $5 \mathrm{mmHg}$ la PAD ${ }^{(30)}$. La ingesta de chocolate oscuro redujo la PAS en 2,9 mmHg y la PAD 1,9 mmHg en 44 personas con pre-hipertensión alta e hipertensión arterial grado $1^{(31)}$.

Como se puede observar en estos pocos estudios clínicos publicados sobre actividad antihipertensiva de las plantas, la presión arterial disminuye menos de $10 \mathrm{mmHg}$. En nuestro estudio, la PAD disminuyó significativamente en $5,33 \mathrm{mmHg}(p<0,05)$ respecto al placebo, al final del experimento. A pesar que la PAS no disminuyó significativamente con el tratamiento con P. edulis (figura 1), se produjo una reducción de $6,73 \mathrm{mmHg}$ (grupo EM4) respecto del placebo, al final del experimento. Esto no deja de ser importante, porque según el sexto reporte del Joint National Committee on Prevention, Detection, Evaluation and Treatment of High Blood Pressure, del National Institutes of Health de los EEUU, estima que una disminución de $5 \mathrm{mmHg}$ produciría una reducción en 14\% de las muertes por apoplejía, un $9 \%$ de reducción de las muertes por enfermedad cardíaca, y un $7 \%$ de reducción en la mortalidad total ${ }^{(32)}$.

En el año 2004, Li y col. ${ }^{(33)}$ demostraron que el flavonoide luteolina, aislado de Cynara scolymus L., incrementó la actividad del promotor de la sintasa del óxido nítrico endotelial y la expresión del ARNm de sintasa del óxido nítrico endotelial, en células endoteliales humanas, con el consecuente incremento de óxido nítrico. Por otra parte, Ko y col. demostraron que la luteolina produjo una inhibición no selectiva de la fosfodiesterasa (PDE1-5), una enzima que hidroliza al AMPc y/o GMPc que tienen un rol importante en la regulación de la vasodilatación ${ }^{(34)}$.

Otros estudios han demostrado el rol de los compuestos fenólicos y flavonoides en la modulación de la reactividad vascular. Así, los compuestos polifenólicos del vino rojo y otras plantas mostraron un efecto vasorrelajante en aorta torácica de rata ${ }^{(35)}$. Los flavonoides del jugo de uvas 
púrpuras incrementaron la liberación de óxido nítrico derivado de plaquetas y disminuyeron la producción de superóxido, un radical libre altamente reactivo que degrada al óxido nítrico ${ }^{(36)}$. También, se ha demostrado que las proantocianidinas pueden inhibir la unión de la angiotensina II al receptor de angiotensina I (AT1) ${ }^{(37)}$. Es conocido el efecto antioxidante de los flavonoides y su capacidad para barrer radicales libres de superóxido y peroxilo y para inhibir una variedad de enzimas responsables de la producción de superóxido ${ }^{(38,39)}$, lo cual es un hecho importante si se tiene en cuenta que el anión superóxido y otras especies reactivas del oxígeno pueden oxidar al óxido nítrico a formas intermediarias más activas, tales como peroxinitrito, las cuales son capaces de producir nitrosilación y/o nitración tóxica de algunos residuos aminoácidos de proteínas, tales como tirosina, produciendo inhibición de la producción de óxido nítrico y/o inactivación del óxido nítrico producido, dañando la función endotelial ${ }^{(40,41)}$. En este sentido, se ha informado que los flavonoides mostraron protección endotelial contra injuria oxidativa ${ }^{(42)}$. Además, se ha demostrado la actividad antioxidante de los polifenoles por incremento de la expresión de catalasa ${ }^{(43)}$.

En el año 2006, se comunicó que un extracto metanólico de la corteza de Passiflora edulis mostró efecto antihipertensivo en ratas espontáneamente hipertensas, y se sugirió que dicho efecto probablemente se debería al ácido $\gamma$-aminobutírico (GABA), dado que se encontró una cantidad relativamente alta de este aminoácido en la corteza ${ }^{(44)}$. Hayakawa y col. demostraron que la administración oral de ácido $\gamma$-aminobutírico disminuyó significativamente la presión arterial sistólica en ratas espontáneamente hipertensas, sugiriendo que el GABA tiene efecto antihipertensivo debido a su inhibición de la liberación de noradrenalina desde los nervios simpáticos en el lecho arterial mesentérico, vía receptores $\mathrm{GABA}_{\mathrm{B}}$ presinápticos ${ }^{(45)}$. Existe la posibilidad que el ácido $\gamma$-aminobutírico encontrado en la corteza de $P$. edulis también se pueda encontrar en el jugo, lo cual contribuiría en parte al efecto antihipertensivo.
Durante el estudio, no se evidenció clínicamente efectos adversos, como náuseas, vómitos, insomnio, mareos, alergia. Solamente en un caso se presentó diarrea leve, pero no motivó la suspensión del tratamiento. Los valores de creatinina, transaminasa glutámica pirúvica y transaminasa glutámica oxalacética y de las demás pruebas realizadas, no mostraron alteración al final del experimento y se mantuvieron dentro de los parámetros considerados normales, lo que nos indica que el tratamiento con el jugo de $P$. edulis no produjo efectos adversos. Maluf y col., $1991^{(46)}$, encontraron que, en un grupo de 9 sujetos sanos que recibieron liofilizado de extracto de la hoja de P. edulis, los valores medios de GOT y amilasa estuvieron incrementados. Esto nos podría indicar que el jugo del fruto de P. edulis, a diferencia del extracto de las hojas, es más tolerable, toda vez que la DL50 para el jugo fue mucho mayor que para las hojas ${ }^{(23)}$. Sin embargo, es necesario llevar a cabo estudios de toxicidad crónica con el jugo para confirmar su seguridad y garantizar su uso en humanos.

Es probable que el efecto antihipertensivo del jugo del fruto de $P$. edulis demostrado en este estudio, se deba a las acciones combinadas de sus diferentes constituyentes químicos que contiene. En el estudio fitoquímico preliminar demostramos en el jugo del fruto la presencia de grupos fenólicos, en especial los flavonoides ${ }^{(47)}$, que confirma la presencia de glicósidos flavonoides como luteolin-6-C-chinovoside y luteolin-6-Cfucoside ${ }^{(48)}$, así como también fenoles ${ }^{(49)}$ y antocianinas ${ }^{(50)}$; estos compuestos contribuirían a reducir la presión arterial con sus propiedades diuréticas, antioxidantes y vasorrelajantes, por incremento de la producción de óxido nítrico ${ }^{(47)}$. El eugenol ${ }^{(51)}$ y triterpenos ${ }^{(52)}$ también podrían contribuir al efecto antihipertensivo. Asimismo, la presencia de ácido ascórbico y caroteno en cantidades relativamente altas en el jugo del fruto ${ }^{(53)}$ serían de mucha importancia para la actividad antioxidante y consecuentemente para el efecto antihipertensivo.

En conclusión, en estas condiciones experimentales, el jugo de Passiflora edulis (maracuyá) fue coadyuvante efectivo del enalapril en la disminución de la presión arterial en pacientes hipertensos en estadio 1, y demostró ser seguro.

\section{AGRADECIMIENTO}

Al Consejo Superior de Investigaciones de la Universidad Nacional Mayor de San Marcos por la financiación del proyecto de investigación.

\section{REFERENCIAS BIBLIOGRÁFICAS}

1. Wolf-Maier K, Cooper R, Banegas J, Giampaoli S, Hense H, Joffres M, et al. Hypertension prevalence and blood pressure levels in 6 European countries, Canada and United States. JAMA. 2003;289:23639.

2. Banegas JR. Epidemiología de la hipertensión arterial en España. Situación actual y perspectivas. Hipertensión. 2005;22(9):353-62.

3. Organización Panamericana de la Salud (OPS). La hipertensión arterial como problema de salud comunitaria. Washington: OPS/OMS; 1984:3.

4. Morales G. Presión arterial sistémica normal y anormal a nivel del mar y la altura. Tesis para optar el grado de Doctor en Medicina. Universidad Peruana Cayetano Heredia. Lima, Perú. 1971.

5. OPS/OMS. Análisis de la situación del Perú. Programa especial de análisis de salud. Lima: Minsa-OPS/OMS; 2002.

6. Agusti R. Epidemiología de la hipertensión arterial en el Perú. Acta Med Per. 2006;23(2):69-75.

7. Katzung B. Farmacología Básica y Clínica. 8a . Ed. México: El Manual Moderno; 2002:181-210.

8. Vila R, Cañigueral S. Fitoterapia de la hipertensión arterial. Módulo 3 Unidad 10. Barcelona: Sandoz Nutrition; 1996:73-7.

9. López M. Las plantas medicinales en el tratamiento de la HTA. Offarm. 2001;20(10):108-10.

10.Zarzuelo A, Duarte J, Jimenez J, González M, Utrilla M. Vasodilatador effect of olive leaf. Planta Med. 1991;57:417-9.

11. Al-Qattan K, Khan I, Alnaqeeb M, Ali M. Mechanism of garlic (Allium sativum) induced reduction of hypertension in $2 \mathrm{~K}-1 \mathrm{C}$ rats: a possible mediation of $\mathrm{Na} / \mathrm{H}$ exchanger isoform-1. Prostaglandins, Leukotrienes and Essential Fatty Acids. 2003;69:217-22.

12. Guerrero M, Puebla P, Carrón R, Martín M, Arteaga L, San Román L. Assessment of the antihypertensive and vasodilator effects of ethanolic extracts of some Colombian medicinal plants. J Ethnopharmacol. 2002;80:37-42.

13. Gilani A, Jabee Q, Ghayur M, Jabanz K, Akhtar M. Studies on the antihypertensive, antispasmodic, bronchodilator and hepatoprotective activities of the Carum copticum seed extract. J Ethnopharmacol. 2005;98:127-35.

14. Yu X, Wu S, Chen C, Pang K, Wong T. Antihypertensive and anti-arrhythmic effects of an extract of Radix Stephaniae tetrandrae in the rat. J Pharm Pharmacol. 2004;56:115-22. 
15. Takei H, Nakai Y, Hattori N, Yamamoto M, Kurauchi $\mathrm{K}$, Sasaki $\mathrm{H}$, et al. The herbal medicine Tokishakayaku-san improves the hypertension and intrauterine growth retardation in preeclampsia rats induced by $\mathrm{N} \omega$-nitro-L-arginine methyl ester. Phytomed. 2004;11:43-50.

16. Negishi H, Xi J, Ikeda K, Njelekela M, Nara Y, Yamori Y. Black and green tea polyphenols attenuate blood pressure increases in stroke-prone spontaneously hipertensive rats. J Nutr. 2004;134:38-42.

17. Duarte J, Pérez-Palencia R, Vargas F, Ocete M, Pérez-Vizcaino F, Zarzuelo A, et al. Antihypertensive effects of the flavonoid quercetin in spontaneously hypertensive rats. Brit J Pharmacol. 2001;133:11724.

18. Jouad H, Lacaille-Dubois M, Lyoussi B, Eddouks M. Effects of the flavonoids extracted from Spergularia purpurea Pers. on arterial blood pressure and renal function in normal and hipertensive rats. J Ethnopharmacol. 2001;76:159-63.

19. García M. Guía Técnica: Cultivo de maracuyá amarillo. El Salvador: Centro Nacional de Tecnología Agropecuaria y Forestal; 2002.

20. Ministerio de Agricultura y Ganadería. Aspectos Técnicos sobre Cuarenta y Cinco Cultivos Agrícolas de Costa Rica. Costa Rica: Dirección General de Investigación y Extensión Agrícola; 1991.

21. Brack A. Diccionario enciclopédico de plantas útiles del Perú. Cusco: Ed. Centro de Estudios Regionales Andinos Bartolomé de las Casas; 1999. p. 36870.

22. Essalud. Formulario Nacional de Recursos Naturales y Afines. Monografía de Plantas Medicinales. Lima: Essalud; p. 204-7.

23. Rojas J, Ronceros S, Palomino R, Tomás G, Chenguayen J. Efecto antihipertensivo y dosis letal 50 del jugo del fruto y del extracto etanólico de las hojas de Passiflora edulis (maracuyá) en ratas. An Fac med. 2006;67(3):206-13.

24. Asociación Médica Mundial. Declaración de Helsinki. Principios éticos para las investigaciones en seres humanos. $54^{\mathrm{a}}$ Asamblea General de la AMM, Tokio. 2004.

25. European Medicines Agengy. Guideline for Good Clinical Practice. ICH Harmonised Tripartite Guideline. CPMP/ICH/135/95. London: EMEA; 2002.

26. Asgary S, Naderi G, Sarrafzadegan N, Mohammadifard N, Mostafavi S, Vaskili R. Antihypertensive and antihyperlipidemic effects of Achillea wilhelmsii. Drugs Exp Clin Res. 2000;26(3):89-93.

27. Park Y, Kim J, Kang M. Concord grape juice supplementation reduces blood pressure in Korean hypertensive men: Double-blind, placebo controlled intervention trial. Biofactors. 2004:22:145-7.

28. Mennen L, Saphinho D, De Bree A, Arnault N, Bertrais S, Galan P, et al. Consumption of foods rich in flavonoids is related to a decreases cardiovascular risk in apparently healthy French women. J Nutr. 2004;134(4):923-6.
29. Duda G, Suliburska J, Pupek-Musialik D. Effects of short-term garlic supplementation on lipid metabolism and antioxidant status in hypertensive adults. Pharmacol Rep. 2008;60:163-70.

30. Perrinjaquet-Moccetti1 T, Busjahn A, Schmidlin C, Schmidt A, Brad B, Aydogan C. Food supplementation with an olive (Olea europaea L.) leaf extract reduces blood pressure in borderline hypertensive monozygotic twins. Phytother Res. 2008:22:1239-42.

31. Taubert D, Roesen R, Lehmann C, Jung N, Schöming E. Effects of low habitual cocoa intake on blood pressure and bioactive nitric oxide. JAMA. 2007;298(1):49-60.

32. National Institutes of Health. National Heart, Lung, and Blood Institute. National High Blood Pressure Education Program. The Sixth Report of the Joint National Committee on Prevention, Detection, Evaluation and Treatment of High Blood Pressure. NIH Publication. No 98-4080. 1997.

33. Li H, Xia N, Brausch I, Yao Y, Forstermann U. Flavonoids from artichoke (Cynara scolymus L.) upregulate endothelial-type nitric-oxide synthase gene expression in human endothelial cells. J Pharmacol Experiment Ther. 2004;310(3):92632.

34. Ko W, Shih Ch, Lai Y, Chen J, Huang H. Inhibitory effects of flavonoids on phosphodiesterase isozymes from guinea pig and their structureactivity relationships. Biochem Pharmacol. 2004;68:2087-94.

35. Andriambeloson E, Magnier C. Natural dietary polyphenolic compounds cause endotheliumdependent vasorelaxation in rat thoracic aorta. J Nutr. 1998;128(12):2324-34.

36. Freedman J, Parker C, Li L, Perlman J, Ivanov V, Deak $\mathrm{L}$. Select flavonoids and whole juice from purple grapes inhibit platelet function and enhance nitric oxide release. Circulation. 2001;103:27928

37. Cos P, De Bruyne T, Hermans N, Apers S, Vanden Berghe D, Vlietinck A. Proanthocyanidins in health care: current and new trends. Current Medicinal Chemistry. 2003;10:1345-59.

38. Woodman 0, Chan E. Vascular and anti-oxidant actions of flavonols and flavones. Clin Experim Pharmacol Physiol. 2004;31:786-90.

39. Nijveldt R, Nood E, Hoorn D, Boelens P, Norren $K$, Leeuwen P. Flavonoids: a review of probable mechanisms of action and potential applications. Am J Clin Nutr. 2001;74:418-25.

40. Achike F, Kwan Ch. Nitric oxide, human diseases and the herbal products that affect the nitric oxide signalling pathway. Clin Experim Pharmacol Physiol. 2003;30:605-15.

41. Gryglewski R, Palmer R, Moncada S. Superoxide anion is evolved in the breakdown of endotheliumderived vascular relaxing factor. Nature. 1986;320:454-6.

42. Vitor R, Mota-Filipe H, Teixeira G, Borges C, Rodrigues $A$, Teixeira $A$, et al. Flavonoids of an extract of Pterospartum tridentatum showing endothelial protection against oxidative injury. J Ethnopharmacol. 2004;93:363-70.

43. Negishi H, Xi J, Ikeda K, Njelekela M, Nara Y, Yamori Y. Black and green tea polyphenols attenuate blood pressure increases in stroke-prone spontaneously hipertensive rats. J Nutr. 2004;134:38-42.

44. Ichimura T, Yamanaka A, Ichiba T, Toyokawa T, Kamada Y, Tamamura T, et al. Antihypertensive effect of an extract of Passiflora edulis rind in spontaneously hypertensive rats. Biosc Biotechnol Biochem. 2006;70(3):718-21.

45. Hayakawa K, Kimura M, Kamata K. Mechanism underlying $\gamma$-aminobutyric acid-induced antihypertensive effect in spontaneously hypertensive rats. Eur J Pharmacol. 2002;438:10713.

46. Maluf E, Barros M, Frochtengarten M, Benti R, Leite J. Assessment of the hypnotic/sedative effects and toxicity of Passiflora edulis aqueous extract in rodents and humans. Phytother Res. 1991;5:262-6.

47. Rojas J. Estudio preclínico y clínico de la seguridad y actividad antihipertensiva de Passiflora edulis Sims (maracuyá). Tesis para optar el Grado de Doctor en Farmacia y Bioquímica. UNMSM. 2009.

48. Mareck U, Herrmann K, Galensa R, Wray V. The 6-C-chinovoside and 6-C-fucoside of luteolin from Passiflora edulis. Phytochem. 1991;30(10):34867.

49. Dhawan K, Dhawan S, Sharma A. Passiflora: a review update. J Ethnopharmacol. 2004;94:123.

50. Kidoy L, Nygard A, Andersen 0, Pedersen A, Aksnes D, Kiremire B. Anthocyanins in fruits of Passiflora edulis and P. suberosa. J Food Compos Analysis. 1997;10(1):49-54.

51. Chassagne D, Crouzet J, Bayonove C, Brillout J, Baumes R. Glycosidically bound eugenol and methyl salicylate in the fruit of edible Passiflora species. J Agricult Food Chem. 1997;45:2685-9.

52. Yoshikawa K, Katsuta S, Mizumori J, Arihara S. Four cycloartane triterpenoids and six related saponins from Passiflora edulis. J Nat Prod. 2000;63(9):1229-34.

53. Pruthi J, Girdhari L. Chemical composition of passion fruit (Passiflora edulis Sims). J Sci Food Agric. 1959;10:188-92.

Manuscrito recibido el 16 de febrero de 2009 y aceptado para publicación el 2 de abril de 2009.

Correspondencia:

Dr. Juan Rojas Armas

Instituto de Investigaciones Clínicas,

Facultad de Medicina - UNMSM

Av. Grau 755. Lima 1, Perú

Correo: jprojasarmas@yahoo.com 